\title{
MANAGEMENT OF COMPLICATED UMBILICAL HERNIA IN CIRRHOTIC PATIENTS
}

\author{
By \\ Mohamed Mohamed Abd El-Kader Megahed, Mohamed Hassan El- \\ Kaseer and Abd El-Salam Amer Emad \\ Department of General Surgery, Faculty of Medicine, Al-Azhar University \\ Corresponding author: Mohamed Hassan El-Kaseer, \\ E-mail: mohamedelkaseer3322@gmail.com
}

\begin{abstract}
Background: Prosthetic mesh reinforcement of abdominal wall hernias has gained acceptance as a result of its ease of placement and a favorably low incidence of hernia recurrence.

Objective: To evaluate the outcome of complicated umbilical hernial repair after herniorrhaphy or hernioplasty in patients with advanced cirrhosis and refractory ascites regarding postoperative morbidity and mortality.

Patients and methods: This study was a prospective randomized controlled study designed for cirrhotic cases presented with complicated umbilical hernia. It was conducted during the period between January 2019 and January 2020 at Al-Azhar University Hospitals. The study included a total of who were randomly divided into two equal groups; Group A 20 cases who underwent herniorrhaphy, and Group B 20 cases who underwent hernioplasty.

Results: CTP class B was present in 65 and $70 \%$ of cases in both groups respectively, while the remaining cases had class $\mathrm{C}$ disease. $\mathrm{CBC}$, liver function, and renal function parameters were not significantly different between the two groups, apart from platelet count, that was significantly higher in group B. The degree of ascites did not significantly differ between the two groups, and ascites was present in all of the included cases. Obstruction was the commonest complication encountered as it was present in $40 \%$ of cases in both groups. Other causes included strangulation, leaking hernia, and irreducibility. The type of complication did not significantly differ between the two groups. Resection anastomosis of a part of the small intestine was performed in 25 and $20 \%$ of cases in both groups respectively. Operative time was significantly longer in group B. Hospital stay was not significantly different between the two groups. The incidence of morbidity and mortality did not significantly differ between the two groups. Recurrence was significantly higher in group A.
\end{abstract}

Conclusion: The use of a prosthetic mesh in complicated cases showed an advantage over the conventional techniques as it was associated with lower recurrence rates with no difference in post-operative morbidity and mortality compared to herniorrhaphy.

Keywords: Complicated Umbilical Hernia, Cirrhosis, Refractory ascites.

\section{INTRODUCTION}

Cirrhosis is common with alcohol abuse and hepatitis virus infection. Raised intraabdominal pressure leads to umbilical hernia in $20 \%$ of those patients (Abbas et al., 2017), in addition to portal hypertension that induce umbilical vein dilatation with widening of the preexistent supra-umbilical fascial opening (Elshoura and Elbedewy, 2019).

Surgery for patient with liver cirrhosis is considered a "difficult field" because of 
many factors, such as deficiency in protein synthesis and coagulation disorders, in addition to respiratory and lung disease or cardiac dysfunction associated with late stages (Bhangui et al., 2012). Complications of umbilical hernias in those patients with cirrhosis and tense refractory ascites include ulceration, leakage, incarceration, and rupture (Triantos et al., 2011).

Flood syndrome, or spontaneous umbilical hernia rupture, is an important complication of longstanding tense ascites and end-stage liver disease. Rupture may follow a sudden increase in intraabdominal pressure such as vomiting, coughing, and straining of stool. The spontaneous rupture and evisceration is usually preceded by other factors such as inflammation that weaken the hernia covering (Good et al., 2011 and Gupta et al., 2011).

Many studies found that the results of surgical repair might depend on the degree of ascites and liver functions (Saleh et al., 2015). Elective umbilical herniorrhaphy is a safe and effective method in a majority of cirrhotic patients in whom ascites is controlled adequately (Eker et al., 2011). However, it is better to be avoided in patients with uncontrolled ascites. There is an absence of high-quality prospective study about management of cirrhotic patients having umbilical hernia to be sure of the right decision (Ammar, 2010).

Indications, time, and technique of herniorrhaphy in such patients remain a matter of controversy. The use of mesh and laparoscopic access is also subject to debate (Hassan et al., 2014). There is an increase in the recurrence rate of umbilical hernia following its correction in cirrhotic patients, and thus hernioplasty with the use of prolene mesh in its repair has been introduced (Holihan et al., 2016).

Previous studies have studied the hernia repair with mesh in comparison with the conventional anatomical repair (herniorrhaphy) and they found that it might reduce the recurrence rate of hernia, but may increase the risk of some complications - e.g. seroma and infection (Umemura et al., 2015).

The technique of mesh repair, i.e., 'hernioplasty', involved either a mesh plug, which is put in the defect, or a flat mesh put over the defect with or without sutures to preserve the mesh secure. The most common mesh used is synthesized from polypropylene prosthetic material. There are many conflicting results on whether the mesh should be used in umbilical hernia repair especially in the complicated settings (Ammar, 2010).

The aim of our study was to evaluate the outcome of complicated umbilical hernial repair after herniorrhaphy or hernioplasty in patients with advanced cirrhosis and refractory ascites regarding postoperative morbidity and mortality.

\section{PATIENTS AND METHODS}

This study was a prospective randomized controlled study designed for cirrhotic cases presented with complicated umbilical hernia. It was conducted during the period between January 2019 and January 2020 at Al-Azhar University Hospitals.

The included patients were divided into two equal groups: Group A underwent herniorrhaphy and Group B underwent hernioplasty. 


\section{Inclusion criteria:}

- Ascitic patients presented with complicated umbilical hernia.

- Leaking umbilical hernia treated with simple sutures at emergency department and re operated after subsidence of inflammation.

- Irreducible umbilical hernia without gangrenous content.

- Strangulated hernia with gangrenous content.

\section{Exclusion criteria:}

- Recurrent cases.

- Patients with advanced coagulopathy and recurrent attacks of hepatic encephalopathy.

- Infected hernias.

Patient consent: A written informed consent was obtained from each patient fulfilling inclusion criteria after full explanation of the operation, its benefits and its possible subsequent complications.

All patients were subjected to the following:

1. Full history taking.

2. Clinical examination.

3. Full preoperative investigations.

After the preoperative evaluation, all patients were classified according to Child-Pugh-Turcotte score (Sumida et al., 2019).

\section{Preoperative preparation}

- Preoperative optimal management of ascites by diuretics (spironolactone and furosemide), early nutritional support, intravenous albumin and /or paracentesis.
- Intravenous antibiotics (e.g. 3rd generation cephalosporin) were prescribed for all cases preoperatively, and these antibiotics were commenced till 3rd post-operative day in patients who did not experience complications. However, in complicated cases, antibiotic administration was continued according to patient condition. Additionally, culture and sensitivity were ordered if needed.

- Fresh frozen plasma and cryoprecipitate were used for correction of preexisting coagulopathy.

\section{Operative steps:}

1. Position: The patient was placed in supine position on the operating table; the arms were positioned at the patient's sides to facilitate access.

2. Incision: Elliptical incision around the hernial sac was performed.

3. Dissection: Identification, dissection of the sac with treatment of the content when necessary, followed by the preparation of the aponeurotic margins.

\section{Repair:}

A. Anatomical repair: Primary umbilical hernial repair was performed by conventional interrupted technique using non-absorbable suture material.

B. Mesh repair: Closure of the abdominal wall defect if possible, followed by application of nonabsorbable mesh prosthesis. This mesh was fixed using non-absorbable suture materials. 
Post-operative follow-up:

\section{A. For early complications patients were followed up 2 and 4 weeks after operation to assess:}

1. Seroma formation.

2. Hematoma.

3. Wound dehiscence.

4. Paralytic ileus.

5. Wound infection.

6. Transient ascites leak.

7. Hepatic coma.

8. Hematemesis.

9. Deterioration of liver function tests.

B. For late complications: Recurrence was assessed every 3-month period for at least 6 months by clinical examination, and abdominal
ultrasonography.

\section{Statistical analysis:}

The collected data were coded, processed and analyzed using the SPSS (Statistical Package for the Social Sciences) version 22 for Windows® (IBM SPSS Inc, Chicago, IL, USA). Data were tested for normal distribution using the Shapiro Walk test. Qualitative data were represented as frequencies and relative percentages. Chi square test $(\chi 2)$ to calculate difference between two or more groups of qualitative variables. Quantitative data were expressed as mean \pm SD (Standard deviation). Independent samples t-test was used to compare between two independent groups of normally distributed variables (parametric data). $\mathrm{P}$ value $<0.05$ was considered significant.

\section{RESULTS}

The mean age of the included cases was 58.6 and 57.93 years in groups A and $B$ respectively. Males represented 75 and $80 \%$ in both groups respectively, while the remaining cases were females. There was no significant difference between the two study groups regarding either of the previous parameters.

Based on CTP classification, 65 and $70 \%$ of cases had class B, whereas the remaining cases had class $C$ in the study groups respectively. There was no significant difference between the two groups regarding CTP score.
$\mathrm{CBC}$, liver function, and renal function parameters were not significantly different between the two groups, apart from platelet count, that was significantly higher in group B (70 vs. $64-\mathrm{p}=0.015$ ).

Pelviabdominal US revealed mild ascites in 40 and $35 \%$ of cases, while moderate ascites was diagnosed in 45 and $55 \%$ of cases in both groups respectively. The remaining cases in both groups had marked ascites. The amount of ascites was not a significant variable when comparing both groups (Table 1). 
Table (1): Demographic data, child Pugh classification, laboratory parameters and Ultrasound findings in the study groups

\begin{tabular}{|l|c|c|c|}
\hline \multicolumn{1}{|c|}{ Groups } & Group A (n= 20) & Group B (n= 20) & $\begin{array}{c}\text { P } \\
\text { value }\end{array}$ \\
\hline Age (years) & $58.6 \pm 3.5$ & $57.93 \pm 4.1$ & $>0.05$ \\
\hline Gender: & $15(75 \%)$ & $16(80 \%)$ & $>0.05$ \\
-Male & $5(25 \%)$ & $4(20 \%)$ & \\
-Female & $13(65 \%)$ & $14(70 \%)$ & $>0.05$ \\
\hline Child Pugh classification: & $7(35 \%)$ & $6(30 \%)$ & $>0.05$ \\
CTP class B & & & \\
CTP class C & $11.21 \pm 2.12$ & $10.95 \pm 2.25$ & $>0.05$ \\
\hline Laboratory parameters: & $9.14 \pm 2.98$ & $9.24 \pm 2.07$ & 0.223 \\
Hemoglobin & $64(29-93)$ & $70(37-102)$ & \\
WBCs & $2.69 \pm 0.5$ & $2.85 \pm 0.48$ & $>0.05$ \\
Platelets & $2.17 \pm 0.83$ & $2.19 \pm 0.71$ & 0.784 \\
Albumin (g/dl) & $58.32 \pm 16.50$ & $59.85 \pm 11.95$ & 0.175 \\
Bilirubin (mg/dl) & $61.86 \pm 16.69$ & $65.36 \pm 12.11$ & 0.114 \\
SGOT (IU/l) & $1.31 \pm 0.35$ & $1.4 \pm 0.41$ & 0.241 \\
SGPT (IU/l) & $1.23 \pm 0.48$ & $1.31 \pm 0.56$ & 0.306 \\
INR & & & \\
Creatinine (mg/dl) & $8(40 \%)$ & $7(35 \%)$ & \\
\hline Ultrasound findings: & $9(45 \%)$ & $11(55 \%)$ & \\
Mild ascites & $3(15 \%)$ & $2(10 \%)$ & \\
Moderate ascites & \multicolumn{3}{|}{} \\
Marked ascites & &
\end{tabular}

Obstruction was the commonest complication encountered as it was present in $40 \%$ of cases in both groups. Other causes included strangulation, leaking hernia, and irreducibility. During operation, resection anastomosis of a part of the small intestine was performed in 25

and $20 \%$ of cases in both groups respectively. Operative time was significantly longer in group B (76.3 vs. 56.7 minutes in both groups respectively $\mathrm{p}=0.001)$. Hospital stay was not significantly different between the two groups $(\mathrm{p}=0.158)($ Table 2$)$.

Table (2): Operative data in the study groups

\begin{tabular}{|l|c|c|c|}
\hline Groups & Group A (n= 20) & Group B (n = & P value \\
\hline Cause of operation & $8(40 \%)$ & $8(40 \%)$ & \\
-Obstruction & $3(15 \%)$ & $2(10 \%)$ & $>0.05$ \\
-Strangulation & $4(20 \%)$ & $6(30 \%)$ & \\
-Leaking & $5(25 \%)$ & $4(20 \%)$ & \\
-Irreducibility & $5(25 \%)$ & $4(20 \%)$ & $>0.05$ \\
\hline Resection anastomosis & $56.7 \pm 10.4$ & $76.3 \pm 8.3$ & $0.001^{*}$ \\
\hline Operative time (minutes) & $4.8 \pm 1.68$ & $5.25 \pm 1.41$ & $>0.05$ \\
\hline Hospital stay & & & \\
\hline
\end{tabular}


As shown in the following table, the incidence of morbidity and mortality did not significantly differ between the two groups $(p>0.05)$. However, recurrence was significantly higher in group A $(45 \%$ vs. $10 \%$ in group $\mathrm{B}-\mathrm{p}=0.001)$. The encountered mortality was due to liver cell failure (Table 3).

Table (3): Post-operative complications

\begin{tabular}{|l|c|c|c|}
\hline Groups & $\begin{array}{c}\text { Group A } \\
(\mathbf{n = 2 0})\end{array}$ & $\begin{array}{c}\text { Group B } \\
(\mathbf{n = 2 0})\end{array}$ & P value \\
\hline Infection & $2(10 \%)$ & $4(20 \%)$ & $>0.05$ \\
\hline Seroma & $2(10 \%)$ & $2(10 \%)$ & 1 \\
\hline Hematoma & $2(10 \%)$ & $1(5 \%)$ & $>0.05$ \\
\hline Transient ascitic leakage & $3(15 \%)$ & $1(5 \%)$ & $>0.05$ \\
\hline Liver function deterioration & $5(25 \%)$ & $5(25 \%)$ & 1 \\
\hline Mortality & $3(15 \%)$ & $3(15 \%)$ & 1 \\
\hline 6-month recurrence & $9(45 \%)$ & $2(10 \%)$ & $0.013 *$ \\
\hline
\end{tabular}

\section{DISCUSSION}

This study was conducted at Al-Azhar University Hospitals aiming to evaluate the outcome of complicated umbilical hernial repair after herniorrhaphy or hernioplasty in patients with advanced cirrhosis and refractory ascites regarding postoperative morbidity and mortality. We included a total of 40 cases that were divided into two equal groups; group A included cases who underwent herniorrhaphy, and group B that included cases who underwent hernioplasty. The mean age of the included cases was 58.6 and 57.93 years in groups $\mathrm{A}$ and $\mathrm{B}$ respectively, with no significant difference between the two groups.

Habib et al. (2017) also reported a mean age of the included cases near to ours, with no significant difference between the two groups. The mean age of the included cases was 56.6 and 56.32 years in both groups respectively.

In addition, another study that included 72 cases and handling the same perspective also reported no significant difference between the two groups regarding patient age. The mean age of the included cases was 50.6 and 51.4 years in both groups respectively (Ammar, 2010).

In our study, males represented 75 and $80 \%$ in both groups respectively, while the remaining cases were females. There was no significant difference between the two groups regarding gender.

Also, Ammar (2010) reported also the higher prevalence of male gender in both groups as they constituted 82.86 and $75.67 \%$ of cases in both groups respectively.

Our results coincide with those of the study by $Y u$ et al. (2015) that included 18 patients, in which the incidence of male patients was $61 \%$.

In the current study, there was no significant difference between the two groups regarding CTP class. On analysis, 65 and $70 \%$ of cases had class B, whereas the remaining cases had class $\mathrm{C}$ in the study groups respectively. The increased prevalence of CTP class B and C, together with the absence of class A could be attributed to the fact that all cases had surgical complications, which increase the 
liability for liver decompensation (Poordad, 2015), and thus CTP score rises.

In 2010, Ammar also reported no significant difference between the two groups regarding CTP score. CTP class B was the commonest class encountered as it was present in 85.71 and $78.38 \%$ of cases in the study groups respectively (Ammar, 2010). Likewise, Habib and his associates confirmed the same findings (Habib et al., 2017). Both of these studies are in accordance with our study findings.

In our study, the amount of ascites was not a significant variable when comparing both groups. Pelviabdominal US revealed mild ascites in 40 and $35 \%$ of cases, while moderate ascites was diagnosed in 45 and $55 \%$ of cases in both groups respectively.

On the contrary, another study reported a significant difference between the two groups regarding ascites. Moderate ascites was diagnosed in 75 and $47.2 \%$ of cases in both groups respectively (Habib et al., 2017). The difference between that study and ours could be attributed to the difference in sample size, patient criteria, cause of cirrhosis, degree of decompensation, and statistical tests performed.

Sonography has been shown to be an accurate preoperative technique in adults for confirming hernias evident on clinical examination, which coincides with our plan in patient preparation as sonography has been done for all cases and is helpful in accurate diagnosis (Rana et al., 2019).

In the current study, obstruction was the commonest complication encountered as it was present in $40 \%$ of cases in both groups. Other causes included strangulation, leaking hernia, and irreducibility. There was no significant difference between the two groups regarding that parameter.

Conversely, Habib et al. (2017) reported a significant difference between the two groups regarding the presentation. Obstruction was the commonest complication in the mesh group (79.2\%), while strangulation was the commonest in the herniorrhaphy group $(60.4 \%)$. Other presentations included irreducibility and rupture. As the previous study was a descriptive non-randomized one, there was an obvious tendency towards the avoidance of mesh application in strangulated and infected cases.

In the current study, operative time was significantly longer in group B (76.3 vs. 56.7 minutes in both groups respectively). The increased operative time was needed for mesh fixation in the hernioplasty group, and that step was of course omitted in the herniorrhaphy group. In our study, Hospital stay was not significantly different between the two groups. It had mean duration of 4.8 and 5.25 days in both groups respectively. Habib and his Colleagues (2017) reported that the length of hospital stay was significantly longer in the herniorrhaphy group (5.89 vs. 3.98 days in the hernioplasty group).

On the other hand, another study reported a significantly longer hospital stay in the hernioplasty group (5.8 vs. 4.4 days in the herniorrhaphy group) (Ammar, 2010). This contradicts with our findings.

In the current study, there was no significant difference between the two hernial repair methods regarding the incidence of both post-operative seroma and hematoma. Seroma was present in 
$10 \%$ of cases, while hematoma was encountered in 10 and $5 \%$ of cases in both groups respectively.

Habib et al. (2017) reported that the incidence of seroma and hematoma did not differ between the two intervention methods. Seroma was encountered in 14.6 and $26.4 \%$ in both groups respectively, whereas hematoma occurred in $3.8 \%$ of cases in the hernioplasty group with no cases in the herniorrhaphy group.

Another study conducted by Ammar (2010) reported that the incidence of hematoma/seroma was 8.5 and $10.8 \%$ of cases in both groups respectively. Like our study, there was no significant difference between the two groups regarding that complication.

In the current study, there was no significant difference between the two groups regarding post-operative ascitic leakage. It occurred in 15 and $5 \%$ of cases in the study groups respectively.

In line with our findings, Ammar (2010) reported that there was no significant difference between the two groups regarding post-operative ascitic fluid leakage. It was encountered in 14.2 and $10.8 \%$ of cases in both groups respectively. Furthermore, another Egyptian study reported that ascitic fluid leakage was encountered in 10.4 and $13.2 \%$ of cases in both groups respectively, with no significant difference between the two groups (Habib et al., 2017).

In our study, surgical site infection was encountered in 10 and $20 \%$ of cases in both groups respectively. Although its incidence increased in the hernioplasty group, there was no significant difference between the two groups.

In another study, although the incidence of surgical site infection did not differ significantly between the two groups, it was more encountered in the hernioplasty group (Ammar, 2010). Moreover, Habib et al. (2017) reported that wound infection occurred in 12.5 and $7.5 \%$ of cases in the same groups respectively.

The surgical site infection (SSI) in other studies of umbilical hernia repair ranged from 1.8 to $19 \%$, depending on the presence or absence or predisposing factors (Asolati et al., 2010 and Farrow et al., 2012). Cirrhosis is considered as a risk factor for SSI (Farrow et al., 2012).

In our study, post-operative deterioration of liver function occurred in $25 \%$ of cases in both groups respectively. Another study reported that encephalopathy occurred in $13.7 \%$ of cases whereas variceal bleeding occurred in $3.9 \%$ of cases (Elshoura and Elbedewy, 2019).

In the current study, recurrence was significantly higher in group A. The rate of recurrence in the present study was similar to that published for umbilical hernia repair, which ranged from 0 to $40 \%$ (Asolati et al., 2010, Kurzer et al., 2010, and Marsman et al., 2011).

Similarly, another study confirmed our findings regarding the increased recurrence without mesh repair. Recurrence was encountered in 14.2 and $2.7 \%$ of cases in both groups respectively (Ammar, 2010). Habib et al. (2017) reported that recurrence was encountered 
in 50 and $9.4 \%$ of cases in the same groups respectively.

In our study, mortality was encountered in $15 \%$ of cases in both groups. This coincides with reports from other series such as Lemmer et al. (2010) (11.1\%), Leonetti et al. (2010) (8.4\%) and Leonetti et al. (2010) (8.4\%).

In complicated cases, a higher mortality rate is expected. Habib et al. (2017) reported that $25 \%$ of cases had mortality in the herniorrhaphy group versus no cases in the hernioplasty group. They attributed that to the complexity of complications, increased prevalence of strangulation, and need for resection in that cases.

\section{CONCLUSION}

The use of a prosthetic mesh in complicated cases showed an advantage over the conventional techniques as it was associated with lower recurrence rates with no difference in post-operative morbidity and mortality compared to herniorrhaphy.

\section{REFERENCES}

1. Abbas N, Makker J, Abbas $\mathbf{H}$ and Balar B. (2017): Perioperative care of patients with liver cirrhosis: a review. Health Services Insights, 10: 1178-83.

2. Ammar S. (2010): Management of complicated umbilical hernias in cirrhotic patients using permanent mesh: randomized clinical trial. Hernia, 14(1): 35-38.

3. Asolati M, Huerta S, Sarosi G, Harmon R, Bell C and Anthony, T. (2010): Predictors of recurrence in veteran patients with umbilical hernia: single center experience. The American Journal of Surgery, 192(5): 627630.

4. Bhangui $P$, Laurent $A$, Amathieu $R$ and Azoulay D. (2012): Assessment of risk for non-hepatic surgery in cirrhotic patients. Journal of Hepatology, 57(4): 874-884.

5. Eker HH, Van Ramshorst G, de Goede B, Tilanus H, Metselaar $H$ and De Man $R$. (2011): A prospective study on elective umbilical hernia repair in patients with liver cirrhosis and ascites. Surgery, 150(3): 542546.

6. Elshoura AA and Elbedewy TA. (2019): Surgical repair of umbilical hernia in cirrhotic patients with ascites: is it safe? The Egyptian Journal of Surgery, 38(1): 52-58.

7. Farrow B, Awad S, Berger DH, Albo D, Lee L and Subramanian A. (2012): More than 150 consecutive open umbilical hernia repairs in a major Veterans Administration Medical Center. The American Journal of Surgery, 196(5): 647-651.

8. Good DW, Royds JE, Smith MJ, Neary PC and Eguare E. (2011): Umbilical hernia rupture with evisceration of omentum from massive ascites: a case report. Journal of Medical Case Reports, 5(1): 170-175.

9. Gupta R, Sah S and Agrawal S. (2011): Spontaneous rupture of incisional hernia: a rare cause of a life-threatening complication. BMJ Case Reports, 11: 486-93.

10. Habib FM, Sallam AM and Gertallah LM. (2017): Mesh or not in the repair of complicated umbilical hernia in cirrhotic patients with decompensated liver cell failure. The Egyptian Journal of Surgery, 36(4): 44046.

11. Hassan AMA, Salama AF, Hamdy H, Elsebae MM, Abdelaziz AM and Elzayat WA. (2014): Outcome of sublay mesh repair in non-complicated umbilical hernia with liver cirrhosis and ascites. International Journal of Surgery, 12(2): 181-185.

12. Holihan JL, Nguyen DH, Nguyen MT, Mo J, Kao LS and Liang MK. (2016): Mesh location in open ventral hernia repair: a systematic review and network meta-analysis. World Journal of Surgery, 40(1): 89-99.

13. Kurzer M, Belsham $P$ and Kark A. (2010): Tension-free mesh repair of umbilical hernia as a day case using local anaesthesia. Hernia, 8(2): 104-107. 
14. Lemmer JH, Strodel WE and Eckhauser FE. (2010): Umbilical hernia incarceration: a complication of medical therapy of ascites. Am J Gastroenterol., 78(5):295-6.

15. Leonetti JP, Aranha GV, Wilkinson WA, Stanley $M$ and Greenlee HB. (2010): Umbilical herniorrhaphy in cirrhotic patients. Archives of Surgery, 119(4): 442-445.

16. Marsman HA, Heisterkamp J, Halm JA, Tilanus HW, Metselaar HJ and Kazemier G. (2011): Management in patients with liver cirrhosis and an umbilical hernia. Surgery, 142(3): 372-375.

17. O'Hara ET, Oliai A, Patek AJ and Nabseth DC. (2010): Management of umbilical hernias associated with hepatic cirrhosis and ascites. Annals of Surgery, 181(1): 85-93.

18. Rana P, D'Souza J, Joshi P, Bandgar A, Mahajan $M$ and Kulthe N. (2019): Dynamic ultrasound in evaluating the spectrum of anterior abdominal wall lesions. International Surgery Journal, 6(7): 2464-2468.

19. Saleh F, Okrainec A, Cleary SP and Jackson TD. (2015): Management of umbilical hernias in patients with ascites: development of a nomogram to predict mortality. The American Journal of Surgery, 209(2): 302-307.

20. Sumida Y, Kamada Y, Iwai M, Kwo PY and Yoneda M. (2019): Laboratory Tests in Liver Diseases Diagnosis of Liver Disease, Pbl. Springer, Pp. 19-34.

21. Triantos CK, Nikolopoulou V, Kehagias I and Burroughs AK. (2011): Surgical repair of umbilical hernias in cirrhosis with ascites. The American Journal of the Medical Sciences, 341(3): 222-226.

22. Umemura A, Suto T, Sasaki A, Fujita T, Endo F and Wakabayashi G. (2015): Laparoscopic umbilical hernia repair in a cirrhotic patient with a peritoneovenous shunt. Asian Journal of Endoscopic Surgery, 8(2): 212-215.

23. Yu BC, Chung $M$ and Lee $G$ (2015): The repair of umbilical hernia in cirrhotic patients: 18 consecutive case series in a single institute. Annals of Surgical Treatment and Research, 89(2): 87-91. 


\section{التدبير العلاجي لحالات الفَتْ السري المعَقّدة في مرضى التليف الكبدي}

محمد محمد عبد القادر مجاهد، محمد حسن القصير، عبد السلام عامر عماد قسم الجراحة العامه، كلية الطب، جامعة الأزهر

E-mail: mohamedelkaseer3322@gmail.com

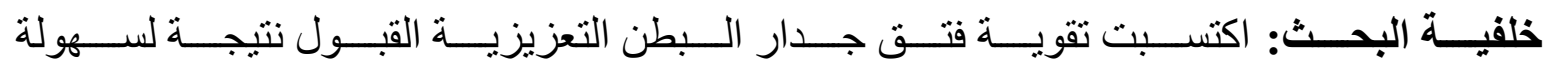

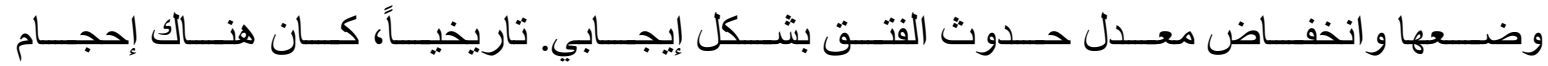

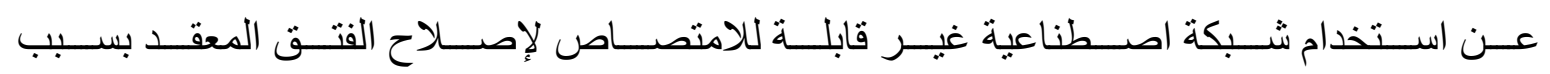

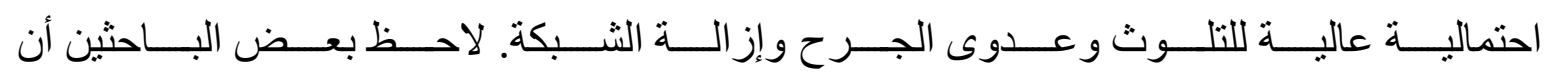

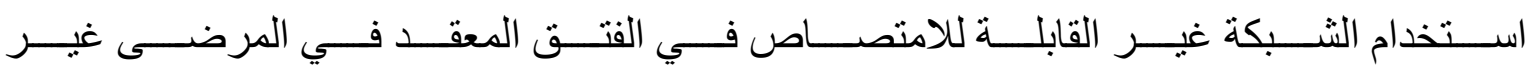

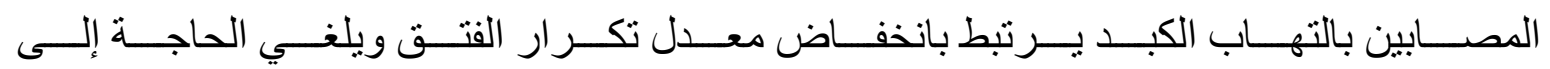
مزيد من الجر احة.

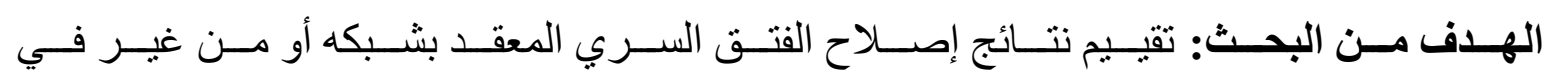

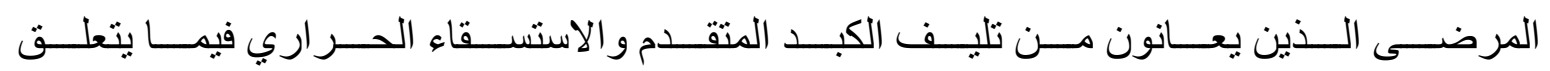
بالمر اضة و الوفيات بعد الجر احة.

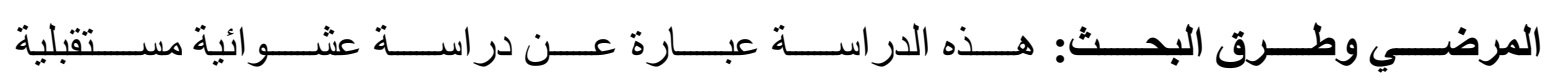

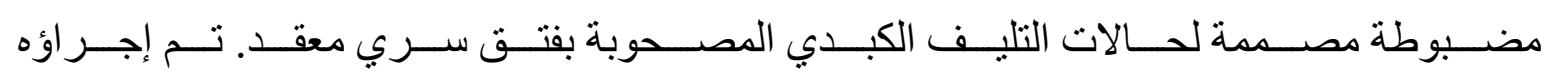

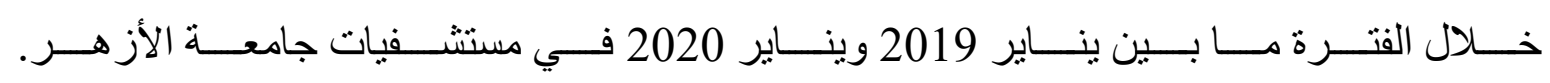

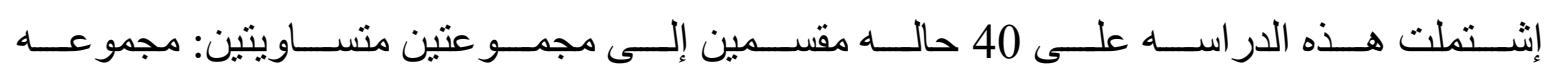
(أ) 20 حالة تم علاجها من غير شبكه. مجموعة (ب) 20 حالة تم علاجها بشبكه.

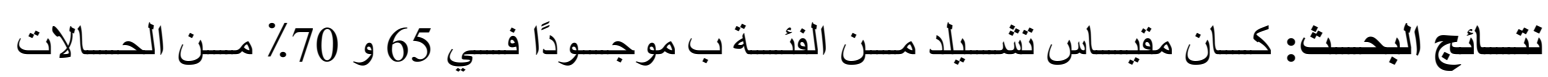

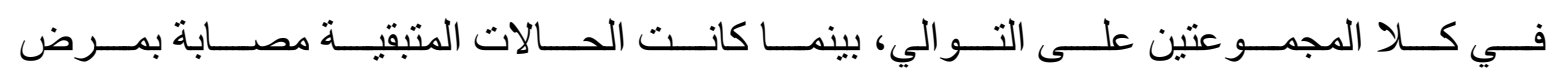

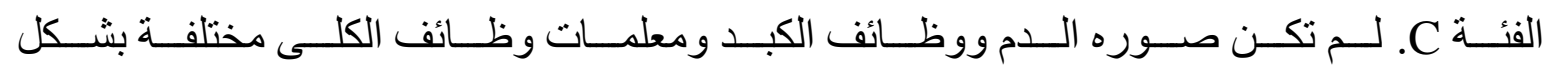

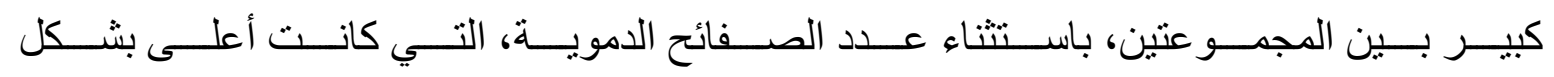

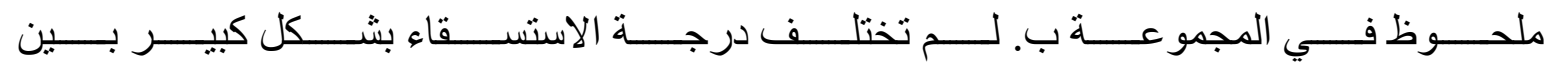

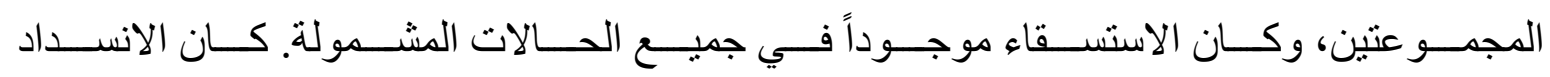




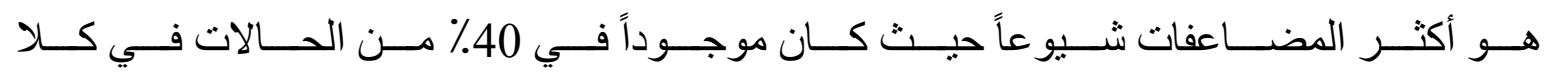

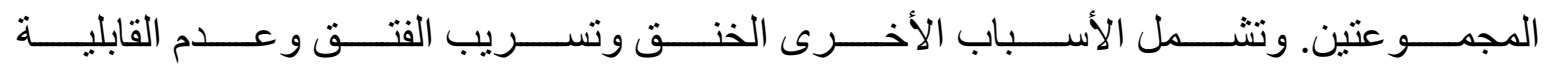

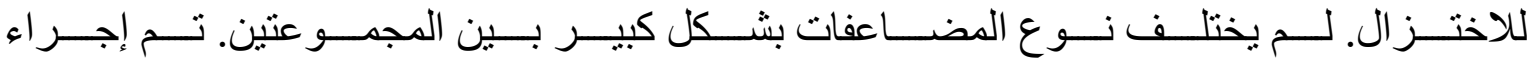

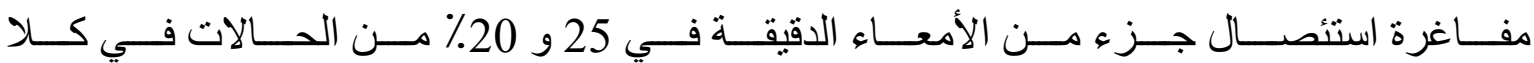

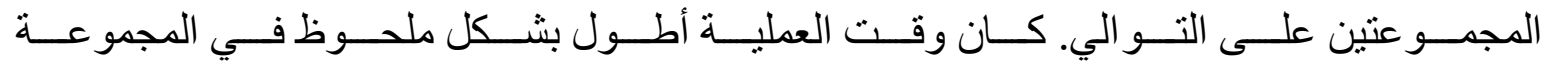

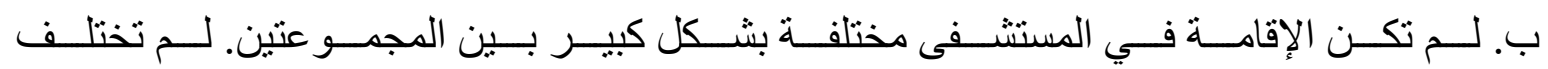

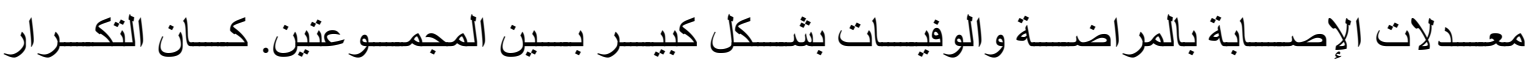
أعلى بشكل ملحوظ في المجمو عة أ.

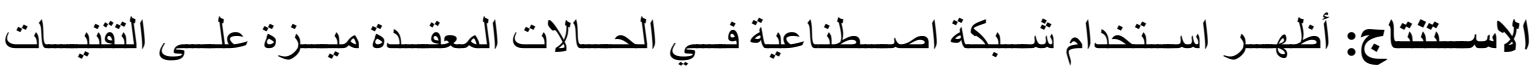

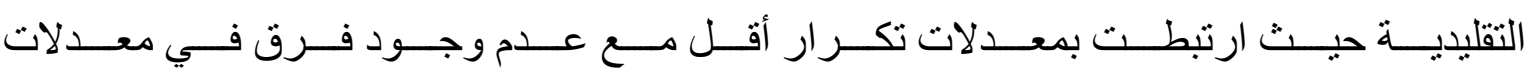
المر اضة و الوفيات بعد الجر احة مقارنة بالفتق. الكلمات الدالة: الفتق السري المعقد، تليف الكبد، الاستسقاء المقاوم للعلاج. 